\title{
BACTERIOLOGICAL PROFILE OF OSTEOMYELITIS CASES WITH SPECIAL REFERENCE TO ANTIBIOTIC SUSCEPTIBILITY PATTERN OF ISOLATES IN A TERTIARY CARE HOSPITAL OF EASTERN INDIA
}

\author{
Anupama Singh ${ }^{1}$, Priyanka Paul Biswas ${ }^{2}$, Aninda Sen ${ }^{3}$ \\ 1 Junior Resident, Department of Microbiology, Katihar Medical College, Katihar. \\ ${ }^{2}$ Assistant Professor, Department of Microbiology, Katihar Medical College, Katihar. \\ 3Professor, Department of Microbiology, Katihar Medical College, Katihar.
}

ABSTRACT
BACKGROUND
Despite advances in medical and surgical therapies, the management of osteomyelitis is an increasing challenge to clinicians due
to growing resistances to antibiotics. The present study was conducted to determine the bacteriological profile of cases of
osteomyelitis and also to ascertain the antibiotic susceptibility pattern of these isolates to prevent unnecessary morbidity and
mortality.

\section{OBJECTIVE}

The proposed study was carried out to isolate, identify, and characterize the bacterial strains isolated from cases of acute and chronic osteomyelitis and to ascertain the antimicrobial susceptibility pattern of these isolates.

\section{MATERIAL AND METHODS}

A total of 97 samples like pus or exudates or pieces of necrotic tissues were collected during surgery if possible, otherwise aspirated. All samples were subjected to Gram staining and culture. Various organisms were identified by standard methods. The Kirby-Bauer method was employed to perform the antimicrobial susceptibility on Mueller-Hinton agar [MHA]. For detection of methicillin-resistant Staphylococcus aureus [MRSA], MHA supplemented with $4 \% \mathrm{NaCl}$ was used.

\section{RESULTS}

A total of 94 organisms were isolated from indoor patient department. Of the unimicrobial isolates, Escherichia coli (19.0\%) were the main organism. Amongst the polymicrobial growth, the common combination was between Staphylococcus aureus and E. coli. The most common factor leading to osteomyelitis was trauma/accidents, 49.5\% (48/97). S. aureus, was the common organism isolated from trauma/accident (83.3\%) cases. Of the total, 30.2\% (16/53) of strains were MRSA. 50\% of MRSA isolates were found in patients with orthopaedic implants. Among the gram-negative bacilli, E. coli showed maximum resistance to amoxicillin (100\%) followed by P. aeruginosa that showed maximum resistance to ciprofloxacin (77.7\%).

\section{CONCLUSION}

Our study will thereby guide the clinicians in choosing appropriate antibiotics, which not only contribute to better treatment, but the judicious use of such antibiotics will also help in preventing emergence of resistance to the drug, which are still sensitive.

\section{KEYWORDS}

Methicillin Resistant Staphylococcus Aureus; Osteomyelitis.

HOW TO CITE THIS ARTICLE: Singh A, Biswas PP, Sen A. Bacteriological profile of osteomyelitis cases with special reference to antibiotic susceptibility pattern of isolates in a tertiary care hospital of eastern India. J Evolution Med Dent Sci. 2016;5(53):34963501, DOI: $10.14260 /$ jemds/2016/807

\section{INTRODUCTION \\ Osteomyelitis is a serious deep bone infection with significant morbidity and high rate of recurrence. The infection caused by a variety of microbial agents can arise from a variety of aetiologies such as trauma, nosocomial infections, or after implant replacement surgery. The future era with modern high speed travel, use of implants, and prosthetics will add to the load of osteomyelitis Treatment of osteomyelitis is challenging particularly when complex multi-drug resistant bacterial biofilm has already been established.}

Financial or Other, Competing Interest: None.

Submission 21-05-2016, Peer Review 14-06-2016,

Acceptance 20-06-2016, Published 02-07-2016.

Corresponding Author:

Dr. Aninda Sen,

Department of Microbiology,

Katihar Medical College,

Karim Bagh, Katihar-854105.

Bihar.

E-mail: aninda_0428@yahoo.com

DOI: $10.14260 /$ jemds $/ 2016 / 807$
Bacteria in biofilm persist in a low metabolic phase causing persistent infection due to increased resistance to antibiotics. ${ }^{1}$

Thus, earlier diagnosis like $\mathrm{x}$-ray and bacterial cultures are needed to prevent unnecessary morbidity and mortality. The other main issue that needs to be addressed during treatment is proper care of wound, debridement of dead tissue, earlier treatment with combination with parenteral and oral antibiotics that will help in reducing the development of resistance among organisms. Since anaerobic bacteria play an important role in chronic osteomyelitis, it should be included in antimicrobial coverage. ${ }^{2}$

There have been no reports of studies on the bacteriological profile of osteomyelitis in and around Katihar/Kosi region. Due to increase in road traffic accidents resulting in compound fractures and also increase in the number of orthopaedic surgeons using implants, iatrogenic and chronic osteomyelitis is being encountered more frequently. ${ }^{1}$ 
Also, due to poverty and illiteracy prevailing in this region, there are more chances of acute osteomyelitis turning into chronic cases and presenting in the hospital. The study was therefore undertaken to determine the bacteriological profile of these cases of osteomyelitis and also to ascertain the antibiotic susceptibility pattern of these isolates that will go a long way in helping the clinician in deciding upon the treatment regime for these patients. The data generated by these studies will also help in formulating hospital antibiotic policies.

\section{MATERIAL AND METHODS}

Study Population: In this study, a total number of 97 cases were studied to ascertain the bacteriological profile of Osteomyelitis in patients admitted in the Department of Orthopaedics of Katihar Medical College for a period of 18 months extending from December 2014 to May 2015 of which 89 showed growths of various organisms and remaining 8 samples were sterile. Informed consent from all cases was taken before collection of samples. The study sought for and obtained ethical clearance from the Institutional Ethics Committee before it was started.

Isolation and Identification: Samples like pus or exudates or pieces of necrotic tissues were collected during surgery if possible, otherwise aspirated. Swabs were collected only when collection during surgery and aspiration was not possible.
Two samples of the same specimen were collected aseptically using sterile cotton swabs. The swabs were transferred to sterile test tubes and transported to the laboratory as soon as possible. ${ }^{3}$

Pus, exudates/swab was inoculated on 5\% Blood agar [BA], Nutrient agar [NA], MacConkey's agar [MA] without crystal violet, and Mannitol salt agar, aerobically at $37^{\circ} \mathrm{C}$ for 24 hours. After incubation, the plates were examined for growth and colony morphology. If the culture showed different types of colonies, subculture was done from single colony to obtain pure growth.

Bacterial growth on BA, NA, MA, and MSA were processed for identification and characterization up to species level. Identification and characterization of gram-negative organisms up to species level were done by a battery of standard tests. ${ }^{3}$

Antimicrobial Susceptibility Testing: Antibiotic sensitivity testing was done by modified Kirby-Bauer's disc diffusion test on Mueller-Hinton agar as per the Clinical and Laboratory Standards Institute [CLSI, 2013]. For detection of MRSA strains, MHA [HiMedia, Mumbai, India] supplemented with $4 \%$ $\mathrm{NaCl}$ was used. Inoculum was prepared and adjusted to 0.5 McFarland's standard. Antibiotic discs were obtained from the HiMedia Laboratories [Mumbai]. ${ }^{4}$ the concentration of each antimicrobial agent [In $\mu \mathrm{g}]$ tested per disc are mentioned in the Table 1.

\begin{tabular}{|c|c|c|c|c|c|}
\hline $\begin{array}{c}\text { Antibiotic Discs for } \\
\text { Gram Positive } \\
\text { Bacteria }\end{array}$ & $\begin{array}{c}\text { Strength } \\
\text { in } \boldsymbol{\mu g}\end{array}$ & $\begin{array}{c}\text { Antibiotic Discs for Gram } \\
\text { Negative Bacteria }\end{array}$ & $\begin{array}{c}\text { Strength } \\
\text { in } \boldsymbol{\mu g}\end{array}$ & $\begin{array}{c}\text { Antibiotic Discs for non- } \\
\text { Fermenters }\end{array}$ & $\begin{array}{c}\text { Strength } \\
\text { in } \boldsymbol{\mu g}\end{array}$ \\
\hline Amoxicillin & 10 & Amoxicillin & 10 & Ceftazidime & 30 \\
\hline Cephalexin & 30 & Cefuroxime & 30 & Piperacillin & 100 \\
\hline Cefoxitin & 30 & Cefotaxime & 30 & Ciprofloxacin & 5 \\
\hline Netilmicin & 10 & Cefoperazone & 75 & Cefoperazone & 75 \\
\hline Ciprofloxacin & 5 & Cefepime & 30 & Cefepime & 30 \\
\hline Erythromycin & 15 & Amikacin & 30 & Amikacin & 30 \\
\hline Amikacin & 30 & Gentamycin & 30 & Gentamycin & 30 \\
\hline Vancomycin & 30 & Ciprofloxacin & 5 & Tobramycin & 10 \\
\hline Linezolid & 30 & Piperacillin/Tazobactam & $100 / 10$ & Piperacillin/Tazobactam & $100 / 10$ \\
\hline Clindamycin & 10 & Cefoperazone/Sulbactam & $75 / 10$ & Cefoperazone/Sulbactam & $75 / 10$ \\
\hline
\end{tabular}

\section{RESULTS}

A total of 97 cases were studied to ascertain the bacteriological profile of Osteomyelitis in patients admitted in the Department of Orthopaedics of Katihar Medical College, of which 89 [91.8\%] showed growth of various organisms and remaining 8 [8.2\%] samples were sterile. Out of the 97 samples, 84 [86.6\%] showed unimicrobial growth and 5 [5.2\%] showed polymicrobial growth. A total of 94 organisms were isolated from indoor patient department.

Out of the total of 94 isolates, $61.7 \%$ were gram-positive organisms including [53 isolates of S. aureus and 5 isolates of CONS] whereas $38.3 \%$ were gram-negative organisms that included [18 isolates of E. coli, 7 isolates of Klebsiella pneumonia, 9 of Pseudomonas aeruginosa, and 2 of Proteus mirabilis]. Of the unimicrobial isolates, Escherichia coli [19.0\%] was the main organism followed by Pseudomonas aeruginosa [8.3\%], Klebsiella pneumoniae and CONS [5.9\% each] and Proteus mirabilis [2.3\%]. Amongst the polymicrobial growth, the common combination was between $S$. aureus and
E. coli. Of the unimicrobial cases, $27.4 \%(23 / 84)$ were found in the age group $16-20$ years followed by $16.7 \%$ (14/84) in 21 25 years and $13.1 \%(11 / 84)$ in 26-30 years. Amongst the polymicrobial cases, $20.0 \%(1 / 5)$ each were found in $21-25$ years followed by 26-30 years and 31-35 years. Most of the sterile growths were in the age group $0-5$ years, $50.0 \%(4 / 8)$.

Out of the 97 cases, $73.2 \%$ (71/97) of patients were males and $26.8 \%$ (26/97) were female. The overall male to female ratio was $2.7: 1$.

The commonest bone affected in the study was tibia $62.9 \%$ (61/97) followed by femur $28.9 \%$ (28/97) and the other small bones (foot and sacrum $3.1 \%$ each and spine $2.1 \%$ ).

Table 4 shows the incidence of predisposing factors in osteomyelitis cases.

The most common factor leading to osteomyelitis was trauma/accidents $49.5 \%$ (48/97) followed by orthopaedic implants 28.9\% (28/97) and postsurgical wound $21.6 \%$ (21/97). 
Of the trauma/accidents cases, $64.6 \%(31 / 48)$ were males and $35.4 \%$ (7/48) were females whereas osteomyelitis due to orthopaedic implants was seen in $78.6 \%(22 / 28)$ males and $21.4 \%(6 / 28)$ of females. Osteomyelitis due to postsurgical wound infection was seen in 85.7\% (18/21) males and 14.3 $(3 / 21)$ females [Table 2].

\begin{tabular}{|c|c|c|}
\hline Predisposing Factors & No. of Cases & Percentage \\
\hline Trauma/accidents & 48 & 49.5 \\
\hline Orthopaedic implants & 28 & 28.9 \\
\hline Postsurgical wounds & 21 & 21.6 \\
\hline Total & $\mathbf{9 7}$ & $\mathbf{1 0 0}$ \\
\hline $\begin{array}{r}\text { Table 2: Distribution of Predisposing } \\
\text { Factors in Osteomyelitis Cases }\end{array}$ \\
\hline
\end{tabular}

S. aureus was the common organism isolated from trauma/accident (83.3\%) cases followed by postsurgical wound infections (96.7\%) and orthopaedic implants (45.4\%). Among the gram-negative organisms, E. coli, K. pneumoniae, and $P$. aeruginosa $(4.7 \%$ each $)$ were isolated from trauma/accident cases whereas E. coli was the main isolate from orthopaedic implants and postsurgical wound infections being $27.3 \%$ and $33.3 \%$. On the other hand, $13.6 \%(3 / 5)$ isolates of CONS were associated with orthopaedic implant infections [Table 3].

\begin{tabular}{|c|c|c|c|}
\hline \multirow{2}{*}{ Microorganisms } & \multicolumn{3}{|c|}{ Predisposing Factors } \\
\cline { 2 - 4 } & Trauma/Accidents & Orthopaedic Implants & Postsurgical Wounds \\
\hline S. aureus & $35(83.3 \%)$ & $10(45.4 \%)$ & $8(96.7 \%)$ \\
\hline CONS & 0 & $3(13.6 \%)$ & $2(6.7 \%)$ \\
\hline E. coli & $2(4.7 \%)$ & $6(27.3 \%)$ & $10(33.3 \%)$ \\
\hline Klebsiella pneumoniae & $2(4.7 \%)$ & $1(4.5 \%)$ & $4(13.3 \%)$ \\
\hline Pseudomonas aeruginosa & $2(4.7 \%)$ & $2(9.1 \%)$ & $5(16.7 \%)$ \\
\hline Proteus mirabilis & $1(2.3 \%)$ & 0 & $1(3.3 \%)$ \\
\hline Total = 94 & $\mathbf{4 2}$ & $\mathbf{2 2}$ & $\mathbf{3 0}$ \\
\hline \multicolumn{4}{|r}{} \\
\hline
\end{tabular}

The study results showed that $69.8 \%(37 / 53)$ of the strains of S. aureus were MSSA and 30.2\% (16/53) of strains were MRSA. Majority of the MRSA (31.3\%) isolates were recovered during increased length of stay in the hospital i.e. $\geq 30$ days whereas $32.5 \%$ MSSA isolates were recovered during the initial period of stay i.e. 11-20 days [Table 4]. In case of MRSA, resistance was seen with amoxicillin (100\%) followed by cephalexin, netilmicin, amikacin, and ciprofloxacin being $62.5 \%$ each [Table 5]. On the other hand, $12.5 \%$ and $6.3 \%$ strains were resistant to vancomycin and linezolid by disc diffusion method. Among the gram-negative bacilli, E. coli showed maximum resistance to amoxicillin (100\%) followed by $50.0 \%$ resistance to cefuroxime, cefotaxime, and cefoperazone each. Klebsiella pneumonia showed 100\% resistance each to amoxicillin, cefuroxime, and cefotaxime. However, only $16.7 \%$ strains of $E$. coli and $42.8 \%$ strains of $K$ pneumonia were resistant to gentamicin [Table 6]. Amongst the non-fermenters, $P$. aeruginosa showed maximum resistance to ciprofloxacin $(77.7 \%)$, piperacillin and cefepime
(55.5\% each), and ceftazidime (33.3\%). Maximum sensitivity was seen with tobramycin, gentamicin $(77.7 \%$ each) followed by amikacin, piperacillin/tazobactam (55.5\%). P. mirabilis were resistant to most of the antibiotics except cefepime, tobramycin, cefoperazone/sulbactam, and cefotaxime [Table 7].

\begin{tabular}{|c|c|c|c|c|}
\hline $\begin{array}{c}\text { Hospital } \\
\text { Stay }\end{array}$ & MRSA & Percentage & MSSA & Percentage \\
\hline $1-10$ & 3 & 18.8 & 7 & 18.9 \\
\hline $11-20$ & 4 & 25.0 & 12 & 32.5 \\
\hline $21-30$ & 4 & 25.0 & 10 & 27.0 \\
\hline$\geq 30$ & 5 & 31.3 & 8 & 21.6 \\
\hline Total & $\mathbf{1 6}$ & $\mathbf{1 0 0}$ & $\mathbf{3 7}$ & $\mathbf{1 0 0}$ \\
\hline \multicolumn{5}{|c|}{ Table 4: Duration of Hospital Stay with Reference to } \\
MRSA/MSSA Infections \\
\hline
\end{tabular}

\begin{tabular}{|c|c|c|c|c|c|c|}
\hline \multirow{3}{*}{ Antibiotics } & \multicolumn{2}{|c|}{$\begin{array}{c}\text { Methicillin Sensitive } \\
\text { S. Aureus (MSSA), n=37 }\end{array}$} & $\begin{array}{c}\text { Methicillin Resistant } \\
\text { S. Aureus (MRSA), no }\end{array}$ & \multicolumn{3}{c|}{ CONS, (n= 5) } \\
\cline { 2 - 7 } & $\begin{array}{c}\text { Sensitive } \\
\mathbf{n}(\%)\end{array}$ & $\begin{array}{c}\text { Resistant } \\
\mathbf{n}(\%)\end{array}$ & $\begin{array}{c}\text { Sensitive } \\
\mathbf{n}(\%)\end{array}$ & $\begin{array}{c}\text { Resistant } \\
\mathbf{n}(\%)\end{array}$ & $\begin{array}{c}\text { Sensitive } \\
\mathbf{n}(\%)\end{array}$ & $\begin{array}{c}\text { Resistant } \\
\mathbf{n}(\%)\end{array}$ \\
\hline Amoxicillin & 0 & $37(100)$ & 0 & $16(100)$ & 0 & $5(100)$ \\
\hline Cephalexin & $7(18.9)$ & $30(81.8)$ & $6(37.5)$ & $10(62.5)$ & $2(40.0)$ & $3(60.0)$ \\
\hline Netilmicin & $10(27.0)$ & $27(72.9)$ & $6(37.5)$ & $10(62.5)$ & $3(60.0)$ & $2(40.0)$ \\
\hline Ciprofloxacin & $7(18.9)$ & $30(81.8)$ & $6(37.5)$ & $10(62.5)$ & $1(20.0)$ & $4(80.0)$ \\
\hline Erythromycin & $15(40.5)$ & $22(59.5)$ & $7(43.8)$ & $9(56.2)$ & $1(20.0)$ & $4(80.0)$ \\
\hline Amikacin & $30(81.0)$ & $7(18.9)$ & $6(37.5)$ & $10(62.5)$ & $3(60.0)$ & $2(40.0)$ \\
\hline Vancomycin & $37(100)$ & 0 & $14(87.5)$ & $2(12.5)$ & $3(60.0)$ & $2(40.0)$ \\
\hline Linezolid & $37(100)$ & 0 & $15(93.8)$ & $1(6.3)$ & $4(80.0)$ & $1(20.0)$ \\
\hline Clindamycin & $30(81.0)$ & $7(18.9)$ & $12(75.0)$ & $4(25)$ & $3(60.0)$ & $2(40.0)$ \\
\hline Gentamicin & $30(81.0)$ & $7(18.9)$ & $7(43.8)$ & $9(56.2)$ & $3(60.0)$ & $2(40.0)$ \\
\hline
\end{tabular}




\begin{tabular}{|c|c|c|c|c|c|c|}
\hline Antibiotics & \multicolumn{3}{|c|}{ Escherichia Coli, n (18) } & \multicolumn{2}{c|}{ Klebsiella Pneumoniae, n (7) } \\
\hline & S & I & R & S & I & R \\
\hline Amoxicillin & 0 & 0 & $18(100 \%)$ & 0 & 0 & $7(100 \%)$ \\
\hline Cefuroxime & 0 & 0 & $18(50.0 \%)$ & 0 & 0 & $7(100 \%)$ \\
\hline Cefotaxime & $3(16.7 \%)$ & $6(33.3 \%)$ & $9(50.0 \%)$ & 0 & 0 & $7(100 \%)$ \\
\hline Cefoperazone & $6(33.3 \%)$ & $3(16.7 \%)$ & $9(50.0 \%)$ & $1(14.2 \%)$ & $1(14.3 \%)$ & $5(71.4 \%)$ \\
\hline Cefepime & $6(33.3 \%)$ & 0 & 0 & $3(42.8 \%)$ & $3(42.9 \%)$ & $1(14.2 \%)$ \\
\hline Amikacin & $9(50.0 \%)$ & $6(33.3 \%)$ & $3(16.7 \%)$ & $4(57.1 \%)$ & 0 & $3(42.8 \%)$ \\
\hline Gentamicin & $15(83.3 \%)$ & 0 & $3(16.7 \%)$ & $4(57.1 \%)$ & 0 & $3(42.8 \%)$ \\
\hline Ciprofloxacin & $3(16.6 \%)$ & 0 & 0 & $1(14.2 \%)$ & $3(42.9 \%)$ & $3(42.8 \%)$ \\
\hline Piperacillin/Tazobactam & $3(16.6 \%)$ & $9(50.0 \%)$ & $6(33.3 \%)$ & $1(14.2 \%)$ & $4(57.1 \%)$ & $2(28.5 \%)$ \\
\hline Cefoperazone/Sulbactam & $12(66.6 \%)$ & 0 & 0 & $1(14.2 \%)$ & $1(14.3 \%)$ & $5(71.4 \%)$ \\
\hline
\end{tabular}

Table 6: Antibiotic Sensitivity/Resistant Pattern of Gram-Negative Bacilli/Fermenters

\begin{tabular}{|c|c|c|c|c|c|c|}
\hline Antibiotics & \multicolumn{2}{|c|}{ Pseudomonas Aeruginosa, n (9) } & \multicolumn{3}{c|}{ Proteus Mirabilis, n (2) } \\
\hline & S & I & R & S & I & R \\
\hline Ceftazidime & $2(22.2 \%)$ & $4(44.4 \%)$ & $3(33.3 \%)$ & $1(50.0 \%)$ & 0 & $1(50.0 \%)$ \\
\hline Piperacillin & $4(44.4 \%)$ & 0 & $5(55.5 \%)$ & $1(50.0 \%)$ & 0 & $01(50.0 \%)$ \\
\hline Ciprofloxacin & $2(22.2 \%)$ & 0 & $7(77.7 \%)$ & $1(50.0 \%)$ & 0 & $1(50.0 \%)$ \\
\hline Cefoperazone & $2(22.2)$ & $4(44.4 \%)$ & $3(33.3 \%)$ & $1(50.0)$ & 0 & $1(50.0 \%)$ \\
\hline Cefepime & $4(44.4 \%)$ & 0 & $5(55.5 \%)$ & $2(100 \%)$ & 0 & 0 \\
\hline Amikacin & $5(55.5 \%)$ & 0 & $4(44.4 \%)$ & $1(50.0 \%)$ & 0 & $1(50.0 \%)$ \\
\hline Gentamicin & $7(77.7 \%)$ & 0 & $2(22.2 \%)$ & $1(50.0 \%)$ & 0 & $1(50.0 \%)$ \\
\hline Tobramycin & $7(77.7 \%)$ & 0 & $2(22.2 \%)$ & $2(100 \%)$ & 0 & 0 \\
\hline Piperacillin/Tazobactam & $5(55.5 \%)$ & $3(33.3 \%)$ & $1(11.1 \%)$ & $1(50.0 \%)$ & 0 & $1(50.0 \%)$ \\
\hline Cefoperazone/Sulbactam & $4(44.4 \%)$ & $4(44.4 \%)$ & $1(11.1 \%)$ & $1(50.0 \%)$ & 0 & 0 \\
\hline Cefotaxime & $4(44.4 \%)$ & $4(44.4 \%)$ & $1(11.1 \%)$ & $0(50.0 \%)$ & 0 & 0 \\
\hline Table 7: Antibiotic Sensitivity/Resistant Pattern of Gram-Negative Bacilli/Non-Fermenters \\
\hline
\end{tabular}

\section{DISCUSSION}

Osteomyelitis is one of the vexing diseases amongst people in developing countries like India due to increase in number of drug-resistant strains that makes treatment even more complicated in addition to requirement of aggressive surgical debridement. Chronic osteomyelitis may require antimicrobial therapy for months to years sometimes with antibiotics that are invaluable for hospital environment such as glycopeptides and carbapenems. Hence, area wise studies on bacteriological profiles and monitoring of antimicrobial susceptibility pattern needs to be carried out in individual settings, which would guide to develop a policy on appropriate use of antibiotics.

Out of the 97 samples, $84(86.6 \%)$ showed unimicrobial growth and $5(5.2 \%)$ showed polymicrobial growth. A total of 94 organisms were isolated from indoor patient department that included (Staphylococcus aureus, CONS, Escherichia coli, Klebsiella pneumoniae, Pseudomonas aeruginosa, and Proteus mirabilis in the decreasing order of isolation). Wadekar DM et al. (2014), reported similar findings where $87.0 \%$ samples were found to be culture positive whereas $13.0 \%$ samples were culture negative; monomicrobial growth was seen in $67.0 \%$ and polymicrobial growth in $20.0 \%$ cases. Collection of specimen before the administration of antibiotics, use of proper transport media, and other factors play a role in incidence of positive culture. 5

Of the unimicrobial growth, isolates were Staphylococcus aureus (58.3\%) followed by Escherichia coli (19.0\%), Pseudomonas aeruginosa (8.3\%), Klebsiella pneumoniae (5.9\%), and Proteus mirabilis (2.3\%). Amongst the polymicrobial growth, most common combination of isolates was between $S$. aureus and E. coli being $40.0 \%$. Kaur J et al. (2008), reported that although bone infections caused by gram-negative organisms had significantly increased, but $S$. aureus (43.0\%) remained the most common cause of osteomyelitis, which was followed by P. aeruginosa (10.0\%),
Proteus spp (6.0\%), Klebsiella spp and E. coli (5.0\% each), Enterobacter spp (3.0\%), S. epidermidis (4.0\%), Streptococcus pyogenes, and Enterococcus spp (2.0\% each). ${ }^{6}$

Out of the 97 cases, $73.2 \%$ (71/97) of patients were males and $26.8 \%$ (26/97) were female. The overall male to female ratio was 2.7:1. Wadekar M et al. (2014), also reported a higher incidence of osteomyelitis in male than in females with the ratio of 2.7:1. The predominance of male patients may point towards gender bias present in the society. This can also be attributed to more exposure to trauma in males. ${ }^{5}$

The commonest bone affected in the study was tibia $62.9 \%$ (61/97) followed by femur $28.9 \%$ (28/97) and the other small bones (foot and sacrum $3.1 \%$ each and foot $2.1 \%$ ). Kaur J et al. (2008), reported similar findings where long bones of the lower extremity were involved in $60 \%$ cases while those of upper extremity were involved in $14.0 \%$ cases. The bones involved in rest of the cases included short bones of hand and feet $(10.0 \%)$, pelvic bones $(8.0 \%){ }^{6}$

The most common factor leading to osteomyelitis was trauma/accidents, 49.5\% (48/97) followed by orthopaedic implants, $28.9 \%$ (28/97) and postsurgical wound, $21.6 \%$ (21/97). Wadekar DM. et al. (2014), reported the common predisposing factor for osteomyelitis to be trauma (44.0\%), which was followed by postsurgical infections $(23.0 \%)$ and orthopaedic implants (21.0\%). 5

Of the trauma/accidents cases, $64.6 \%$ (31/48) were males and $35.4 \%(7 / 48)$ were females whereas osteomyelitis due to orthopaedic implants was seen in $78.6 \%(22 / 28)$ males and $21.4 \%(6 / 28)$ of females. Osteomyelitis due to postsurgical wound infection was seen in $85.7 \%(18 / 21)$ males and 14.3 (3/21) females. Suguneswari G et al. (2013), reported that majority of accident cases was seen in males $71.6 \%(38 / 53)$ as compared to female $28.3 \%(15 / 53)$. 
Similarly, $84.6 \% \quad(22 / 26)$ and $100 \% \quad(19 / 19)$ of postsurgical wound infections and prosthesis induced infections were seen in male patients. ${ }^{7}$

S. aureus, was the common organism isolated from trauma/accident (83.3\%) cases followed by postsurgical wound infections (96.7\%) and orthopaedic implants (45.4\%). Among the gram-negative organisms, E. coli, K. pneumoniae, and $P$. aeruginosa $(4.7 \%$ each $)$ were isolated from trauma/accident cases whereas $E$. coli was the main isolate from orthopaedic implants and postsurgical wound infections being $27.3 \%$ \& $33.3 \%$. On the other hand, $13.6 \%$ (3/5) isolates of CONS were associated with orthopaedic implant infections.

Gilmore et al. (2009), found that the most common pathogen responsible for osteomyelitis in humans is Staphylococcus species followed by Enterobacteriaceae and Pseudomonas species. ${ }^{8}$ Chihara S et al. (2010), reported that coagulase-negative staphylococci are often seen in association with foreign bodies such as prosthetic joints. ${ }^{9}$ Contrasting results were shown by Agarwal AC et al. (2008), where E. coli was the main pathogen in $34.4 \%$ cases especially in open fractures, chronic osteomyelitis, bedsores, and patients with spinal instrumentation. ${ }^{10}$ Authors say that E. coli is a commensal of the gut and as many orthopaedic patients are bedridden for prolonged periods, contamination of wounds, dressings, linen, clothes, and even hands during perineal hygiene plays a major role in increasing chances of transmission of infection.

Majority of the MRSA (31.3\%) isolates were recovered during increased length of stay in the hospital i.e. $\geq 30$ days whereas $32.5 \%$ MSSA isolates were recovered during the initial period of stay i.e. 11-20 days. Thus, the number of MRSA strains increased proportionately with increase in the length of stay in hospital due to increased exposure to hospital milieu that harbours the resistant organisms.

Majority of the MSSA showed resistance to amoxicillin followed by cephalexin and ciprofloxacin (100\%, 81.8\%) whereas in case of MRSA resistance was seen with amoxicillin $(100 \%)$ followed by cephalexin, netilmicin, amikacin, and ciprofloxacin being $62.5 \%$ each. Of the MRSA, $12.5 \%$ and $6.3 \%$ strains were resistant to vancomycin and linezolid by disc diffusion method. In case of CONS $100 \%$, resistance was seen with amoxicillin followed by $60.0 \%$ resistance with cephalexin and cefoxitin each. Ali M et al. (2014), reported that all the MSSA strains were susceptible to vancomycin, gentamicin, teicoplanin, ciprofloxacin, and linezolid; while the MRSA strains were sensitive to vancomycin and linezolid. ${ }^{11}$

In other studies, the MRSA isolates showed resistance to commonly used antibiotics like cefepime (100\%), erythromycin (90.9\%), tetracycline (90.9\%), co-trimoxazole (90.9\%), piperacillin/tazobactam $(81.8 \%)$, ciprofloxacin (72.7\%), and levofloxacin (54.5\%). However, all the MRSA strains showed $100 \%$ sensitivity to vancomycin and $91.6 \%$ sensitivity to levofloxacin. ${ }^{7}$ It is quite clear from the studies that have been conducted so far as well as from the present study that MRSA strains are becoming alarming because of their increased resistance towards antibiotics-like amikacin, netilmicin, and to a lesser extent to vancomycin and linezolid that leaves the clinicians with less choice to use the appropriate drug for treatment of chronic osteomyelitis.

In our study, E. coli showed maximum resistance to amoxicillin (100\%) followed by $50.0 \%$ resistance to cefuroxime, cefotaxime, and cefoperazone each. Klebsiella pneumoniae showed $100 \%$ resistance each to amoxicillin, cefuroxime, and cefotaxime. On the other hand, only $16.7 \%$ strains of E. coli and $42.8 \%$ strains of $K$. pneumoniae were resistant to gentamicin. Other authors reported that E. coli and K. pneumoniae showed that $100 \%$ and $80.0 \%$ resistance to ampicillin. A total of $83.8 \%$ strains of gram-negative bacilli were found to be resistant to gentamicin. The authors reported cefoperazone-sulbactam combination and amikacin to be the most effective drug for treating gram-negative organisms. ${ }^{6}$

In our study, $P$. aeruginosa showed maximum resistance to ciprofloxacin (77.7\%), piperacillin and cefepime (55.5\% each), and ceftazidime and cefoperazone being (33.3\%) each. Maximum sensitivity was seen with tobramycin, gentamicin (77.7\% each) followed by amikacin, piperacillin/tazobactam (55.5\%). In other studies, $60.0 \%$ strains of $P$. aeruginosa were found to be resistant to ceftriaxone followed by $58.0 \%$ strains resistant to cefotaxime and $54.0 \%$ each being resistant to cefepime and imipenem. On the other hand, authors reported aztreonam and levofloxacin to be the most active drugs against non-fermenters. ${ }^{5}$

Fifty percent strains of $P$. mirabilis were found to be resistant to ceftriaxone, piperacillin, ciprofloxacin, cefoperazone, amikacin, gentamicin, piperacillin/tazobactam whereas $100 \%$ sensitivity was seen with cefepime, tobramycin, cefoperazone/sulbactam, and cefotaxime. Antibiotic susceptibility pattern in case of $P$. mirabilis as reported by other authors showed maximum resistance to cefotaxime $(75.0 \%)$ followed by ceftazidime and ceftriaxone (63.0\% each) whereas the strains retained $100 \%$ sensitivity to imipenem. ${ }^{5}$

\section{CONCLUSION}

The present study highlights the importance of microbiological examination of bone in cases of osteomyelitis. Microorganisms could not be detected in $8.2 \%$ of cases. The absence of growth may be due to anaerobic organisms, which flourish well in the dead tissues due to lack of oxygen supply. However, $86.6 \%$ of cases showed unimicrobial and $5.2 \%$ had polymicrobial aetiologies. Thus, any bacteria gram-positive or gram-negative either or alone or as mixed infection could be responsible for osteomyelitis.

MRSA isolation in this region was found to be slightly lower (30.2\%) of all Staphylococcus aureus isolates as compared to the isolation rates in some other parts of India, which is probably because the medical college is situated in the rural area where the organisms are not exposed to as much antibiotic pressure as in the urban areas. Thus, the number of MRSA strains increased proportionately with increase in the length of stay in hospital due to increased exposure to the hospital milieu that harbours the resistant organisms. To prevent hospital acquired infection, prolonged hospital stay should be restricted in orthopaedic wards.

Our study revealed that overall gentamicin was the most sensitive drug among all gram-negative bacilli followed by amikacin, tobramycin, and cefepime, most of the gramnegative bacilli were found to be resistant to third generation cephalosporins like cefotaxime, cefuroxime, and ceftazidime. It is important to undertake studies to see the prevalence of bacterial isolates and their antibiotic sensitivity pattern in a particular geographical area that will enable appropriate and judicious selection of antibiotics and would limit the emerging 
drug-resistant strains in the future to treat the disease successfully.

\section{REFERENCES}

1. Gomes D, Pereiera M, Bettencourt AF. Osteomyelitis: an overview of antimicrobial therapy. Br J of Pharmaceutical Sciences 2013;49(1):13-22.

2. Berbari EF, Steckelberg JM, Osmon. Osteomyelitis. In: Mandell GL, Douglas JE, Dolin R. eds. Bennett's principles and practices of infectious diseases. $7^{\text {th }}$ edn. Vol. 1 Philadelphia: Churchill Livingstone 2006:1457-1459.

3. Collee JG, Miles RS, Watt B. Test for identification of bacteria. In: Colles JG, Fraser AG, Marmion BP, et al. eds. Mackie and McCartney practical medical microbiology. 14th edn. New Delhi: Churchill Livingstone 2006:131-149.

4. CLSI. Performance standards for antimicrobial susceptibility testing: $18^{\text {th }}$ informational supplement. M 100-S18, Wayne, PA, USA 2008;28(1).

5. Wadekar MD, Anuradha K, Venkatesh D. Chronic osteomyelitis: aetiology and antibiotic susceptibility pattern. Int J Recent Trends in Science and Technology 2014;9(3):337-340.
6. Kaur J, Gulati VL, Aggarwal A, et al. Bacteriological profile of osteomyelitis with special reference to staphylococcus aures. Indian J for the practicing doctors 2008;4(6):1-9.

7. Suguneswari G, Singh AH, Basu R. Bacteriological profile of osteomyelitis in a tertiary care hospital at Visakhapatnam, Andhra Pradesh. Int J Cur Res and Rev 2013;(20):52-58.

8. Gilmour WM. Acute haematogenous osteomyelitis. J Bone Joint Surg 2009;(4):841-851.

9. Chihara S, Popovich KJ, Weinstein RA, et al. Staphylococcus aureus bacteriuria as a prognosticator for outcome of staphylococcus aureus bacteraemia: a case control study. BMC Infect Disease 2010;10(1):225.

10. Agarwal AC, Jain S, Jain RK, et al. Pathogenic bacteria in an orthopaedic hospital. J Infect Developing Countries 2008;2(2):120-123.

11. Ali M, Kumar R. Evaluation of bacteriological profile of chronic osteomyelitis in a tertiary care hospital. Int J Scientific Research 2014;(3):383-385. 